\title{
Long-Term Patterns of Oral Anticancer Agent Adoption, Duration, and Switching in Patients With CML
}

\author{
Matthew P. Banegas, PhD, MPHa; Donna R. Rivera, PharmD, MScb; Maureen C. O'Keeffe-Rosetti, MSa; \\ Nikki M. Carroll, MSc; Pamala A. Pawloski, PharmDd; David C. Tabano, PhDc,e; Mara M. Epstein, ScDf, ; \\ Kai Yeung, PharmD, PhD ${ }^{\text {; }}$ Mark C. Hornbrook, PhDa; Christine Lu, MSc, PhDij; and Debra P. Ritzwoller, PhD
}

\begin{abstract}
Background: Oral tyrosine kinase inhibitors (TKIs) have been the standard of care for chronic myeloid leukemia (CML) since 2001. However, few studies have evaluated changes in the treatment landscape of CML over time. This study assessed the long-term treatment patterns of oral anticancer therapies among patients with CML. Methods: This retrospective cohort study included patients newly diagnosed with CML between January 1, 2000, and December 31, 2016, from 10 integrated healthcare systems. The proportion of patients treated with 5 FDA-approved oral TKI agents-bosutinib, dasatinib, imatinib, nilotinib, and ponatinib-in the 12 months after diagnosis were measured, overall and by year, between 2000 and 2017. We assessed the use of each oral agent through the fourthline setting. Multivariable logistic regression estimated the odds of receiving any oral agent, adjusting for sociodemographic and clinical characteristics. Results: Among 853 patients with CML, $81 \%$ received an oral agent between 2000 and 2017 . Use of nonoral therapies decreased from $100 \%$ in 2000 to $5 \%$ in 2005 , coinciding with imatinib uptake from 65\% in 2001 to $98 \%$ in 2005. Approximately $28 \%$ of patients switched to a second-line agent, $9 \%$ switched to a third-line agent, and $2 \%$ switched to a fourth-line agent. Adjusted analysis showed that age at diagnosis, year of diagnosis, and comorbidity burden were statistically significantly associated with odds of receiving an oral agent. Conclusions: A dramatic shift was seen in CML treatments away from traditional, nonoral chemotherapy toward use of novel oral TKIs between 2000 and 2017. As the costs of oral anticancer agents reach new highs, studies assessing the long-term health and financial outcomes among patients with CML are warranted.
\end{abstract}

J Natl Compr Canc Netw 2019;17(10):1166-1172 doi: 10.6004/jncen.2019.7303

\footnotetext{
${ }^{a}$ Center for Health Research, Kaiser Permanente Northwest, Portland, Oregon; ${ }^{b}$ Division of Cancer Control and Population Sciences, National Cancer Institute, Bethesda, Maryland; 'Kaiser Permanente Colorado Institute for Health Research, Denver, Colorado; ${ }^{\mathrm{d} H e a l t h P a r t n e r s ~ I n s t i t u t e, ~ B l o o m i n g t o n, ~}$ Minnesota; ${ }^{e}$ Denver Public Health, Denver, Colorado; ${ }^{f}$ Meyers Primary Care Institute, Worcester, Massachusetts; ${ }^{9}$ University of Massachusetts Medical School, Worcester, Massachusetts; ${ }^{h}$ Kaiser Permanente Washington Health Research Institute, Seattle, Washington; 'Harvard Medical School, Boston, Massachusetts; and ${ }^{\mathrm{j} H}$ arvard Pilgrim Health Care Institute, Boston, Massachusetts.
}

\section{Background}

In 2018, approximately 8,430 patients were diagnosed with chronic myeloid leukemia (CML) and 1,090 patients died of the disease in the United States. ${ }^{1}$ Treatment advances in CML using targeted oral therapies have led to dramatic improvements in prognosis, with a 50\% decrease in the mortality rate from 2000 to $2015 .^{2}$ The life expectancy of patients with CML is approaching that of the general population. ${ }^{3}$ Imatinib, an orally administered tyrosine kinase inhibitor (TKI), was approved by the FDA in 2001 as a first-line therapy for CML in adults. However, resistance to imatinib is well-documented, with nearly $20 \%$ of patients displaying primary resistance (initial refractoriness to imatinib) and another $20 \%$ presenting with secondary, or acquired, resistance (developing during imatinib treatment). ${ }^{4}$ To overcome mutational resistance, 3 second-generation oral TKIs - dasatinib, nilotinib, and bosutinib-and 1 thirdgeneration oral TKI, ponatinib, have received approval for use as first-line treatments or salvage therapy options following the use of prior agents. ${ }^{5}$ These 5 oral agents have become the standard of care and compose a complex treatment landscape for patients with CML.

Although prior studies have assessed the use of and patient adherence to oral TKIs to treat $\mathrm{CML}^{6-12}$ few have evaluated the entire scope of treatment patterns among patients with this disease, including use of non-TKI oral therapies, use of nonoral therapies, and nonreceipt of therapy. ${ }^{13,14}$ Current guidelines recommend indefinite TKI treatment for some patients with $\mathrm{CML},{ }^{15}$ although evidence has suggested that imatinib, dasatinib, or nilotinib can safely be discontinued in those with sustained molecular response. ${ }^{16,17}$ Nevertheless, the optimal sequential use of specific TKIs is unclear due to similarities in their mechanisms of action, particularly in the third-line setting and beyond. ${ }^{18}$ Furthermore, research describing the use of sequential treatment regimens for CML in community practice has been limited.

See JNCCN.org for supplemental online content. 
To address this knowledge gap, we used tumor registry data linked to comprehensive electronic health record (EHR), insurance claims, and pharmacy data from 10 large US healthcare systems to assess treatment use patterns, switching, duration, and adherence among adult patients with CML. To our knowledge, this is the first study to assess multimodality treatment use and to provide detailed information on treatment-switching patterns across all FDAapproved oral anticancer agents among patients with CML.

\section{Methods}

\section{Study Setting and Data Sources}

This retrospective analysis of oral and nonoral cancer therapy use was conducted using data from patients diagnosed with CML who were enrolled health plan members and who received care at 1 of 10 integrated health systems (Kaiser Permanente Colorado, Kaiser Permanente Northern California, Kaiser Permanente Northwest, Kaiser Permanente Hawaii, Kaiser Permanente Washington, Reliant Medical Group/Meyers Primary Care Institute, Marshfield Clinic Health System, Geisinger Health System, Henry Ford Health System, and HealthPartners Institute). These systems are members of the NCI-funded Cancer Research Network (CRN), a consortium within the Health Care Systems Research Network (HCSRN).

The HCSRN's Virtual Data Warehouse (VDW) was the primary data source for this study. ${ }^{19-21}$ The VDW is a series of standardized databases and content areas that include enrollment, demographic, tumor registry, pharmacy, vital sign, diagnosis, procedure, infusion, and benefit data. This VDW is populated with data on services received for all patients who receive care via EHR and claims data. Within the VDW, the Virtual Tumor Registry (VTR) contains data consistent with the North American Association of Central Cancer Registries standards. ${ }^{22}$ VTR data were obtained via chart review by trained abstractors and included data associated with health service use and clinical, treatment, and tumor characteristics. The VDW pharmacy files provided data on national drug code-based prescription drugs dispensed from both outpatient pharmacies and infusion centers. Death information was obtained from the VTR, membership files, state-level death registries, and Social Security Administration data.

\section{Study Population}

The study population comprised 853 patients diagnosed with CML (ICD-O-3 codes 9863, 9875, 9876) between January 1, 2000, and December 31, 2016. Eligibility was limited to patients whose CML was their first cancer diagnosis (sequence 00 or 01 ) and who were aged $\geq 21$ years at diagnosis, survived $\geq 1$ month after diagnosis, and were continuously enrolled in the health plan in the 12 months after diagnosis. The follow-up period was defined as January 1, 2000, through December 31, 2017.

\section{Study Measures}

The primary outcome measure was the proportion of patients with CML treated with any of the 5 FDA-approved oral TKIs (bosutinib, dasatinib, imatinib, nilotinib, ponatinib) in the 12 months after diagnosis. In addition, we assessed the proportion of patients who received non-TKI oral agents (ruxolitinib, thalidomide, lenalidomide, chlorambucil, ibrutinib), nonoral therapy, or no cancer therapy. Among patients who received an oral therapy agent (TKI or non-TKI), we calculated the proportion of use of each agent through the fourth-line setting. First-line therapy was defined as the first oral anticancer agent initiated within the first 12 months after diagnosis. Patients were classified as switching (to a second-, third-, and fourth-line therapy) if they had evidence of cessation of the initial agent (ie, no further prescriptions ordered or dispensed for that agent) followed by initiation of a different oral agent (ie, dispensing of another oral agent).

Date of treatment initiation was defined as the first date a patient received an oral agent, as measured by the initial dispensing date for a given agent. Duration of each distinct line of therapy was measured as the time between the date of treatment initiation (day of first dispensing) and the end date. The end date for each line of therapy was defined as the earliest of the following dates: treatment discontinuation, plan disenrollment, death, the day before the first dispensing date of the new therapy (only for those who switched), or the end of the study period (December 31, 2017). The date of discontinuation was defined by the last day of medication availability based on days supplied, before a gap in therapy coverage of $\geq 60$ days. Patients had to be enrolled in the health plan for the entire 60-day gap (unless dropout was due to death) to be identified as discontinuing. Medication adherence was measured using the proportion of days covered (PDC), defined as the total days' supply of agent divided by the duration of each distinct line of therapy. ${ }^{7,23}$ Time to treatment was defined as the number of days between CML diagnosis and treatment initiation (for first-line therapy); for those who switched therapy, it was defined as the number of days between the end date of the previous therapy and date of initiation for the subsequent line of therapy.

\section{Statistical Analysis}

Descriptive statistics were used to characterize baseline patient variables and oral cancer agent use. Baseline patient variables included age at diagnosis, sex, race, ethnicity, study site, year of diagnosis, vital status at the end of the followup period, the Quan adaptation of the Charlson-Deyo comorbidity score (eg, excluding cancer diagnosis), ${ }^{24}$ and duration of follow-up. We calculated the proportions of patients who did and did not receive any oral cancer agent, overall and by year.

For those who received an oral agent, we calculated the proportion who received each type of agent, overall 
and by year, during the study period, and the proportion who received each type of oral agent by line of therapy. We described the most commonly used therapeutic sequence of oral agents received by patients by the total number of agents in the sequence.

Multivariable logistic regression was used to estimate the likelihood of receiving any oral agent, adjusting for age at diagnosis, sex, race, ethnicity, study site, year of diagnosis, Charlson-Deyo comorbidity score, and recipt of hydroxyurea. All statistical tests assumed a 2-sided alpha of .05. Analyses were conducted using SAS 9.4 (SAS Institute, Inc.).

\section{Results}

Among all patients with CML, $81 \%$ were treated with an oral agent (Table 1). Compared with those who did not receive an oral agent, patients who received oral therapy were more likely to be younger $(70 \%$ aged $<65$ years at diagnosis vs $36 \%$ ), nonwhite ( $24 \%$ vs $18 \%$ ), Hispanic ( $12 \%$ vs $6 \%$ ), diagnosed after 2005 ( $74 \%$ vs $53 \%$ ), and have a Charlson-Deyo comorbidity score of 0 ( $63 \%$ vs $42 \%)$.

Figure 1 shows the swift decrease in use of nonoral therapies among patients with CML, from $100 \%$ in 2000 to $5 \%$ in 2005 and continuing thereafter (to $<1 \%$ by 2017), alongside the rapid uptake of oral TKI agents following their FDA approval. In 2001, approximately $65 \%$ of patients received imatinib, increasing to $98 \%$ by 2005 . Use of imatinib began decreasing after 2005, from $97 \%$ in 2006 to $64 \%$ in 2017. Following FDA approval of dasatinib in 2006, patient use of this agent increased from $4 \%$ in 2006 to $26 \%$ by 2015 . Similarly, use of nilotinib increased from $1 \%$ in 2007 when it was approved to $17 \%$ in 2016. However, use of bosutinib remained near $1 \%$ between its FDA approval in 2012 and 2017, and ponatinib use remained at $\leq 1 \%$ following its approval in 2013 .

Among patients who received an oral agent as firstline therapy, $91 \%$ used imatinib, $6 \%$ used dasatinib, $2 \%$ used nilotinib, and $1 \%$ used other oral agents (Table 2). Time to initiation of first-line therapy was shortest for those who received dasatinib (mean, 22.9 days), and the duration of first-line therapy was longest for patients who received imatinib (mean, 47 months). The PDC of firstline therapy was similar across oral agents, ranging from 0.85 for nilotinib to 0.93 for dasatinib. Approximately $28 \%$ of patients who received first-line therapy switched to a second-line oral agent, among which the most commonly used second-line agent was dasatinib (66\%) followed by nilotinib (30\%) and imatinib (3\%); bosutinib was used by $<6$ patients in the second-line setting. Time-to-initiation of second-line therapy was shortest for imatinib (mean, 17.5 days), and the duration of secondline therapy was longest for those who received dasatinib (mean, 28 months).

Nearly 1 in every 3 patients who received second-line therapy switched to a third-line agent, among which

\begin{tabular}{|c|c|c|c|}
\hline Characteristic & $\begin{array}{l}\text { Received } \\
\text { Oral Agent } \\
\text { n (\%) }\end{array}$ & $\begin{array}{l}\text { Did Not Receive } \\
\text { Oral Agentc } \\
\text { n (\%) }\end{array}$ & $P$ Value ${ }^{d}$ \\
\hline Total, n & 694 & 159 & \\
\hline Age at diagnosis & & & $<.0001$ \\
\hline $18-39 y$ & $124(17.9)$ & $13(8.2)$ & \\
\hline $40-64$ y & $358(51.6)$ & $44(27.7)$ & \\
\hline $65-79$ y & $174(25.1)$ & 53 (33.3) & \\
\hline$\geq 80 y$ & $38(5.5)$ & $49(30.8)$ & \\
\hline Mean age (SD), y & $55.9(15.6)$ & $67.5(17.5)$ & $<.0001$ \\
\hline Sex & & & .71 \\
\hline Male & $404(58.2)$ & $90(56.6)$ & \\
\hline Female & $290(41.8)$ & $69(43.4)$ & \\
\hline Race & & & .004 \\
\hline Asian & $67(9.7)$ & $6(3.8)$ & \\
\hline Black & $62(8.9)$ & $10(6.3)$ & \\
\hline $\begin{array}{l}\text { American Indian/Alaskan } \\
\text { Native/Native Hawaiian/ } \\
\text { Pacific Islander }\end{array}$ & $16(2.3)$ & $<6^{\mathrm{e}}(0.6)$ & \\
\hline Other/Unknown & $21(3.0)$ & $12(7.6)$ & \\
\hline White & $528(76.1)$ & $130(81.8)$ & \\
\hline Ethnicity & & & .08 \\
\hline Hispanic & $81(11.7)$ & $9(5.7)$ & \\
\hline Non-Hispanic/Unknown & $613(88.3)$ & $150(94.3)$ & \\
\hline Year of CML diagnosis & & & $<.0001$ \\
\hline 2000-2004 & $179(25.8)$ & $74(46.5)$ & \\
\hline 2005-2009 & $209(30.1)$ & $36(22.6)$ & \\
\hline 2010-2014 & $252(36.3)$ & $42(26.4)$ & \\
\hline $2015-2017$ & $54(7.8)$ & $7(4.4)$ & \\
\hline \multicolumn{2}{|c|}{ Charlson-Deyo comorbidity score ${ }^{f}$} & & $<.0001$ \\
\hline 0 & $438(63.1)$ & $67(42.1)$ & \\
\hline 1 & $128(18.4)$ & $40(25.2)$ & \\
\hline$\geq 2$ & $128(18.4)$ & $52(32.7)$ & \\
\hline $\begin{array}{l}\text { Mean Charlson-Deyo } \\
\text { comorbidity score (SD) }\end{array}$ & $0.8(1.3)$ & $1.3(1.61)$ & $<.0001$ \\
\hline $\begin{array}{l}\text { Mean duration of follow-up } \\
\text { (SD), mo }\end{array}$ & $63.9(49.3)$ & $33.7(43.1)$ & $<.0001$ \\
\hline Received hydroxyurea & $278(40.1)$ & $57(35.9)$ & .33 \\
\hline
\end{tabular}

Abbreviation: $\mathrm{CML}$, chronic myeloid leukemia.

aTable estimates based on 853 patients diagnosed with CML between

2000-2016 who were enrolled health plan members and received care at 1 of 10 integrated health systems.

bOral agents included bosutinib, chlorambucil, dasatinib, ibrutinib, imatinib, lenalidomide, nilotinib, ponatinib, ruxolitinib, and thalidomide.

cNonoral agents included asparaginase, azacytidine, bacille Calmette-Guérin, bendamustine, bevacizumab, busulfan, cetuximab, cyclophosphamide, cytarabine, daunorubicin, decitabine, docetaxel, doxorubicin, etoposide, fludarabine, fluorouracil, gemcitabine, idarubicin, interferon alfa-2a, interferon alfa-2b, interferon beta- $1 a$, melphalan, mitoxantrone, pegaspargase, pentostatin, rituximab, tocilizumab, topotecan, and vincristine.

${ }^{\mathrm{d} B a s e d}$ on chi-square test (for categorical variables) and $t$ test (for continuous variables).

eHIPAA and data use agreements with participating sites prohibit the reporting of exact values when $<6$ patients (but at least 1 patient) had the given variable value. 'Excludes all cancer diagnoses from the index. 


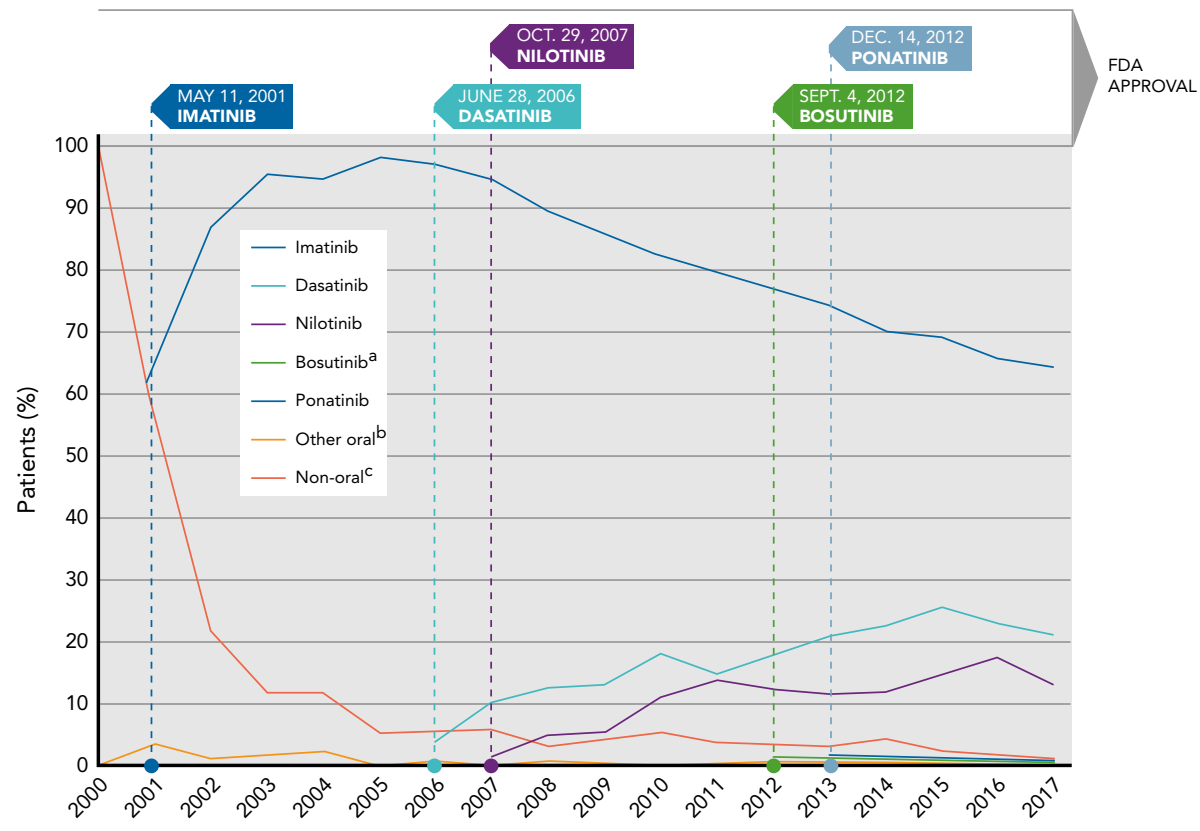

Figure 1. Trends in treatment use among patients with chronic myeloid leukemia.

aPercentages in any given year may not add to $100 \%$ because patients may have used $>1$ therapy in a year.

bOther oral agents included ruxolitinib, thalidomide, lenalidomide, chlorambucil, and ibrutinib.

'Nonoral therapy included asparaginase, azacytidine, bacille Calmette-Guérin, bendamustine, bevacizumab, busulfan, cetuximab, cyclophosphamide, cytarabine, daunorubicin, decitabine, docetaxel, doxorubicin, etoposide, fludarabine, fluorouracil, gemcitabine, idarubicin, interferon alfa-2a, interferon alfa-2b, interferon beta-1a, melphalan, mitoxantrone, pegaspargase, pentostatin, rituximab, tocilizumab, topotecan, and vincristine.

nilotinib was most common (50\%), followed by imatinib (28\%) and dasatinib (9\%); bosutinib and ponatinib were each used by $<6$ patients in the third-line setting. Patients who received nilotinib as third-line therapy had the shortest time to initiation and longest duration of use, whereas those who received dasatinib had the highest PDC (0.98). Approximately $27 \%$ of patients who received third-line therapy switched to fourth-line therapy, with dasatinib the most commonly used fourth-line agent (35\%); each of the remaining agents was used by $<6$ patients.

The most common oral therapy sequences for patients who received up to 4 lines of therapy are presented in Table 3. Among patients who received first-line therapy only, $65 \%$ used imatinib as the first-line agent, $4 \%$ used dasatinib, and $1 \%$ used nilotinib. For patients who received 2 lines of oral therapy $(n=196)$, the most common sequence was imatinib followed by dasatinib (43\%), then imatinib followed by nilotinib (17\%), and dasatinib followed by nilotinib (4\%). Among those who received 3 lines of oral therapy $(n=64), 39 \%$ received imatinib/dasatinib/nilotinib and $11 \%$ received imatinib/ dasatinib/imatinib, with $<6$ patients receiving imatinib/ nilotinib/imatinib or imatinib/nilotinib/dasatinib. Among the patients who received a fourth-line oral therapy $(n=17)$, the 3 most common sequences were imatinib/dasatinib/ imatinib/dasatinib, imatinib/dasatinib/nilotinib/dasatinib, and imatinib/nilotinib/imatinib/nilotinib.
In multivariable regression analysis, the age at diagnosis and Charlson-Deyo comorbidity score were statistically significantly associated with receiving an oral agent (supplemental eTable 1, available with this article at JNCCN.org). Specifically, patients who were older (odds ratio [OR], 0.96; 95\% CI, 0.95-0.97; $P<.0001)$ and those who had a greater comorbidity burden $(\mathrm{OR}, 0.87 ; 95 \% \mathrm{CI}$, 0.76-0.99; $P=.039$ ) had lower odds of receiving an oral agent than those who were younger and had fewer comorbidities. Year of CML diagnosis was statistically significantly associated with greater odds of receiving an oral agent, with patients diagnosed in more recent years having greater odds than those diagnosed earlier (OR, 1.16; 95\% CI, $1.10-1.21 ; P<.0001)$. Sex, race, ethnicity, and receipt of hydroxyurea were not found to be significantly associated with receipt of an oral agent in this population.

\section{Discussion}

This study is the first to quantify the dramatic shift in treatment practices away from nonoral chemotherapy agents toward use of novel oral TKIs in a large cohort of patients treated for CML. In addition, we detailed patterns of switching and multiple lines of use for most patients who initiated oral therapy. The patterns of oral agent use underscore the long treatment duration among many patients with CML (as long as 48 months for imatinib) and the heterogeneity in the sequential use of different oral 


\begin{tabular}{|c|c|c|c|c|c|c|}
\hline Line of Therapy & Imatinib & Dasatinib & Bosutinib & Nilotinib & Ponatinib & Other $^{\mathrm{a}}$ \\
\hline First-line $(n=694), n(\%)$ & $630(90.7)$ & $41(5.9)$ & N/A & $14(2.0)$ & N/A & $9(1.3)$ \\
\hline Time to first-line therapy (mean/median), $d$ & $67.1 / 12$ & $22.9 / 12$ & & $228.6 / 51.5$ & & $476.6 / 91$ \\
\hline Duration (mean), mo & 46.72 & 20.92 & & 23.72 & & 3.85 \\
\hline PDC (mean) & 0.88 & 0.93 & & 0.85 & & 0.91 \\
\hline Second-line ( $n=196), n(\%)$ & $6(3.1)$ & $130(66.3)$ & $<6^{\mathrm{b}}$ & $59(30.1)$ & N/A & N/A \\
\hline Time to second-line therapy (mean/median), $d$ & $17.5 / 0$ & $97.2 / 3.5$ & $0 / 0$ & $121.5 / 6$ & & \\
\hline Duration (mean), mo & 22.36 & 27.74 & 22.16 & 25.96 & & \\
\hline PDC (mean) & 0.91 & 0.84 & 1.00 & 0.83 & & \\
\hline Third-line $(n=64), n(\%)$ & $18(28.1)$ & $6(9.4)$ & $<6^{\mathrm{b}}$ & $32(50)$ & $<6^{\mathrm{b}}$ & $\mathrm{N} / \mathrm{A}$ \\
\hline Time to third-line therapy (mean/median), $d$ & $20.28 / 32.5$ & $15.05 / 11.5$ & $15.47 / 4.5$ & $27.22 / 10.5$ & $4.5 / 0$ & \\
\hline Duration (mean), mo & 116.4 & 26.8 & 173.3 & 22.8 & 39 & \\
\hline PDC (mean) & 0.81 & 0.98 & 0.88 & 0.82 & 0.85 & \\
\hline Fourth-line $(n=17), n(\%)$ & $<6^{\mathrm{b}}$ & $6(35.3)$ & $<6^{\mathrm{b}}$ & $<6^{\mathrm{b}}$ & $<6^{\mathrm{b}}$ & $<6^{\mathrm{b}}$ \\
\hline Time to fourth-line therapy (mean/median), $d$ & $149 / 149$ & $16.8 / 13.5$ & $705.6 / 443$ & $31.7 / 16$ & $18.5 / 18.5$ & $398 / 398$ \\
\hline Duration (mean), mo & 4.30 & 25.72 & 5.89 & 26.70 & 15.21 & 1.67 \\
\hline PDC (mean) & 1.00 & 0.87 & 0.91 & 0.65 & 0.98 & 1.00 \\
\hline
\end{tabular}

Abbreviations: CML, chronic myeloid leukemia; PDC, proportion of days covered.

a Other oral agents included ruxolitinib, thalidomide, lenalidomide, chlorambucil, and ibrutinib.

bHIPAA and data use agreements with participating sites prohibit the reporting of exact values when $<6$ patients (but at least 1 patient) had the given variable value.

agents, with some patients receiving 4 lines of therapy. These findings from real-world community oncology settings address important questions, demonstrating the widespread adoption of new anticancer agents after their FDA approval and the substitution effects on the use of older therapies.

In this analysis, $81 \%$ of patients with newly diagnosed CML received an oral agent between 2000 and 2017, with $99 \%$ of these patients receiving a TKI as their first-line agent. These estimates are higher than those previously reported in the few studies that have assessed nonuse of TKIs. ${ }^{13,14}$ Using SEER-Medicare data, Winn et al ${ }^{13}$ found that approximately $68 \%$ of eligible patients newly diagnosed with CML between 2007 and 2011 initiated TKI therapy within 180 days after diagnosis. Darkow et al, ${ }^{14}$ using pharmacy and medical claims data from 45 large US employers, reported that $42 \%$ of patients newly diagnosed with CML between 1997 and 2009 initiated TKI therapy. Although differences in study design and population preclude a direct comparison across studies, the high proportion reported in our study is based on estimates for patients diagnosed with CML via our tumor registry data linked to actual dispensing data. These data reflect contemporary treatment patterns for all 5 oral TKI agents currently FDA-approved as treatment options within our community practices. Our cohort of patients who received oral agents was mostly aged $<65$ years $(70 \%)$ and had a mean age of 56 years, and therefore higher estimates may be caused by prescribing practices when physicians treated patients with fewer chronic conditions. Additionally, patients with more comorbid conditions were less likely to receive an oral anticancer agent, potentially related to greater physician or patient concerns about adverse events or toxicities.

Consistent with prior studies assessing the use of oral TKI agents in CML treatment across healthcare settings, imatinib was the predominant therapy used between 2001 and 2017,,$^{6-12}$ overall and as first-line therapy. Patients treated with imatinib also had the longest duration of therapy because it was the earliest TKI drug approved. Notably, our study extends this prior research by showing the rapid decline in use of older, nonoral therapies between approximately 2000 and 2005 (from 100\% to 5\%, respectively), coinciding with the prompt uptake of imatinib from $65 \%$ (2001) to $98 \%$ (in 2005) - a clear substitution effect. We also observed a decrease in imatinib use beginning in 2006 (following FDA approval of dasatinib), a finding that reflects trends similar to those shown in a recent study by Cole and Dusetzina, ${ }^{12}$ who reported decreased use of imatinib as a first-line therapy from approximately $100 \%$ between 2001 and 2005 to nearly 40\% in 2014. Although imatinib use did not decrease to $<60 \%$ in our study, these results collectively show a shift over time away from imatinib in the first-line setting toward dasatinib and nilotinib.

Our findings regarding the therapeutic sequences among patients with CML reflect long-term patterns of oral TKI use in community oncology practice and add to a paucity of literature, ${ }^{25-30}$ particularly outside the clinical trial setting., ${ }^{7,10,11}$ Nearly $28 \%$ of patients who initiated a firstline oral agent switched to a second-line agent, consistent 
Table 3. Most Common Oral Therapy Sequences

\begin{tabular}{|c|c|c|c|c|c|}
\hline Line of Therapy & $\begin{array}{l}\text { Patients Who Received } \\
\text { Agent(s) in Sequence } \\
\text { n }\end{array}$ & \multicolumn{4}{|c|}{ Sequence of Agents in Treatment Regimen } \\
\hline \multirow[t]{3}{*}{ First-line $(n=694)$} & 454 & Imatinib & & & \\
\hline & 25 & Dasatinib & & & \\
\hline & 10 & Nilotinib & & & \\
\hline \multirow{2}{*}{ Second-line $(n=196)$} & 84 & Imatinib & Dasatinib & & \\
\hline & 7 & Dasatinib & Nilotinib & & \\
\hline \multirow[t]{4}{*}{ Third-line $(n=64)$} & 25 & Imatinib & Dasatinib & Nilotinib & \\
\hline & 7 & Imatinib & Dasatinib & Imatinib & \\
\hline & $<6^{\mathrm{a}}$ & Imatinib & Nilotinib & Imatinib & \\
\hline & $<6^{\mathrm{a}}$ & Imatinib & Nilotinib & Dasatinib & \\
\hline
\end{tabular}

Table estimates based on the 694 patients diagnosed with CML between 2000 and 2016 who received any oral anticancer agent. The table is not exhaustive and only provides the 3 most common agents or sequences (or 4 if there were equal percentages of patients who received 2 sequences) for each line of therapy. Abbreviation: CML, chronic myeloid leukemia.

aHIPAA and data use agreements with participating sites prohibit the reporting of exact values when $<6$ patients (but at least 1 patient) had the given variable value.

with findings from prior observational studies. ${ }^{7,10,11}$ This finding is also consistent with expected secondary resistance estimates for imatinib requiring a therapeutic switch. ${ }^{31}$ Dasatinib was the most common second-line agent used (66\%) and nilotinib the most common thirdline agent (50\%), indicating that imatinib/dasatinib (for first- to second-line) and imatinib/dasatinib/nilotinib (for first- to third-line) were the specific sequences. These findings are consistent with prior research,, $, 26-28,30$ follow the sequential drug approval years, and highlight the patterns of sequential oral TKI agents in real-world practice.

This study has certain limitations that warrant consideration when interpreting results. First, the study was conducted among patients with cancer enrolled in 10 integrated health systems with standardized prescribing practices, and the findings are most generalizable to similar populations; however, there are also strengths to this population, including the stability of the population over time and access to comprehensive tumor registry and EHR data. Second, we did not have access to data on presence of the BCR-ABLI mutation, phase of CML, or cytogenic or molecular response to therapy, and thus we could not evaluate the association of these factors with therapy initiation, treatment discontinuation, delays in therapy, or switching. Third, we did not have access to data on reasons for discontinuation or switching; however, we relied on the availability of and ability to access comprehensive pharmacy, utilization, infusion, clinical, and demographic information from the EHRs of 10 healthcare systems and report findings from a large sample size. Fourth, in estimating adherence with PDC, we relied on pharmacy data regarding medication dispensing, which does not guarantee that patients took the medication as directed and may lead to an overestimation of adherence. However, pharmacy records are considered to be more accurate than self-reported medication use. ${ }^{32,33}$ Finally, we did not assess patient cost-sharing associated with oral TKI use, although this has been shown to be associated with treatment delays and discontinuation. ${ }^{34}$

\section{Conclusions}

This study provides a comprehensive and longitudinal analysis of the treatment patterns among patients with CML across 10 large US healthcare systems. Our findings highlight the rapid uptake of oral TKI agents over an 18-year period during which 5 novel agents received FDA approval for the treatment of CML. The unique nature of CML and the approaches to treatment, characterized by extended treatment periods that may last the remainder of patients' lives, present opportunities for future research to understand the primary reasons for treatment discontinuation and switching, and to assess the value of therapy for patients and the healthcare system. As the costs of oral anticancer medications continue to increase, ${ }^{12,35}$ studies are warranted to assess the long-term health and financial outcomes among patients with CML and whether the continued benefits outweigh the risks of therapy.

Submitted December 18, 2018; accepted for publication March 29, 2019.

Author contributions: Study concept: Banegas, Rivera, Pawloski, Tabano, Epstein, Ritzwoller. Data analysis: Banegas, Rivera, Pawloski, O'Keeffe-Rosetti, Carroll, Ritzwoller. Data interpretation: All authors. Manuscript writing: All authors. 
Disclosures: Dr. Banegas has disclosed that he has received grant/ research sup-port from AstraZeneca for work that is outside the scope of this study. Dr. Tabano has disclosed that he is employed by Bristol-Myers Squibb. The remaining authors have not received any financial consideration from any person or orga-nization to support the preparation, analysis, results, or discussion of this article.
Funding: This work was supported by an NCl grant (U24CA171524). The content is solely the responsibility of the authors and does not necessarily represent the official views of the $\mathrm{NIH}$.

Correspondence: Matthew P. Banegas, PhD, MPH, Center for Health Research, Kaiser Permanente Northwest, 3800 North Interstate Avenue, Portland, OR 97227-1110. Email: Matthew.P.Banegas@kpchr.org

\section{References}

1. Siegel RL, Miller KD, Jemal A. Cancer statistics, 2018. CA Cancer J Clin 2018;68:7-30

2. Noone AM, Howlader N, Krapcho M, et al, eds. SEER Cancer Statistics Review, 1975-2015, National Cancer Institute. Bethesda, MD. Available at: https://seer.cancer.gov/archive/csr/1975_2015/. Based on November 2017 SEER data submission, posted to the SEER website, April 2018. Accessed May 2, 2019.

3. Bower H, Björkholm M, Dickman PW, et al. Life expectancy of patients with chronic myeloid leukemia approaches the life expectancy of the general population. J Clin Oncol 2016;34:2851-2857.

4. Rogers G, Hoyle M, Thompson Coon J, et al. Dasatinib and nilotinib for imatinib-resistant or -intolerant chronic myeloid leukaemia: a systematic review and economic evaluation. Health Technol Assess 2012;16:1-410.

5. Radich JP, Deininger M, Abboud CN, et al. NCCN Clinical Practice Guidelines in Oncology: Chronic Myeloid Leukemia. Version 4.2018. To view the most recent version, visit NCCN.org. Accessed July 17, 2018.

6. Darkow T, Henk HJ, Thomas SK, et al. Treatment interruptions and non-adherence with imatinib and associated healthcare costs: a retrospective analysis among managed care patients with chronic myelogenous leukaemia. Pharmacoeconomics 2007;25:481-496.

7. Henk HJ, Woloj M, Shapiro M, et al. Real-world analysis of tyrosine kinase inhibitor treatment patterns among patients with chronic myeloid leukemia in the United States. Clin Ther 2015;37:124-133.

8. Rashid N, Koh HA, Lin KJ, et al. Real world treatment patterns in chronic myeloid leukemia patients newly initiated on tyrosine kinase inhibitors in an U.S. integrated healthcare system. J Oncol Pharm Pract 2018;24:253-263.

9. Kreys ED, Frei CR, Villarreal SM, et al. Evaluation of long-term chronic myeloid leukemia treatment practices with tyrosine kinase inhibitors in a national cohort of veterans. Pharmacotherapy 2017;37:278-286.

10. Lang AS, Mounier M, Roques M, et al. A retrospective study of the prescribing and outcomes of tyrosine kinase inhibitors in chronic myeloid leukaemia over a period of more than 10 years. J Clin Pharm Ther 2015;40:391-397.

11. Haque R, Shi J, Chung J, et al. Medication adherence, molecular monitoring, and clinical outcomes in patients with chronic myelogenous leukemia in a large HMO. J Am Pharm Assoc 2017;57:303-310.

12. Cole AL, Dusetzina SB. Generic price competition for specialty drugs: too little, too late? Health Aff (Millwood) 2018;37:738-742.

13. Winn AN, Keating NL, Dusetzina SB. Factors associated with tyrosine kinase inhibitor initiation and adherence among Medicare beneficiaries with chronic myeloid leukemia. J Clin Oncol 2016;34:4323-4328.

14. Darkow T, Maclean R, Joyce GF, et al. Coverage and use of cancer therapies in the treatment of chronic myeloid leukemia. Am J Manag Care 2012;18(Suppl 11):S272-278.

15. Bisen A, Claxton DF. Tyrosine kinase targeted treatment of chronic myelogenous leukemia and other myeloproliferative neoplasms. Adv Exp Med Biol 2013;779:179-196.

16. Rea D, Nicolini FE, Tulliez M, et al. Discontinuation of dasatinib or nilotinib in chronic myeloid leukemia: interim analysis of the STOP 2G-TKI study. Blood 2017;129:846-854.

17. Etienne G, Guilhot J, Rea D, et al. Long-term follow-up of the French Stop Imatinib (STIM1) study in patients with chronic myeloid leukemia. J Clin Oncol 2017:35:298-305.

18. Jabbour E, Kantarjian H, Cortes J. Use of second- and third-generation tyrosine kinase inhibitors in the treatment of chronic myeloid leukemia: an evolving treatment paradigm. Clin Lymphoma Myeloma Leuk 2015;15: 323-334.
19. Ross TR, Ng D, Brown JS, et al. The HMO Research Network virtual data warehouse: a public data model to support collaboration. EGEMS (Wash DC) $2014 ; 2: 1049$

20. Chubak J, Ziebell R, Greenlee RT, et al. The Cancer Research Network: a platform for epidemiologic and health services research on cancer prevention, care, and outcomes in large, stable populations. Cancer Causes Control 2016;27:1315-1323.

21. Hornbrook MC, Hart G, Ellis JL, et al. Building a virtual cancer research organization. J Natl Cancer Inst Monogr 2005;2005:12-25.

22. North American Association of Central Cancer Registries. Strategic management plan: blueprint for action 2011-2016. Available at: https:// www.naaccr.org/wp-content/uploads/2016/11/NAACCR-StrategicManagement-Plan-2011-2016.pdf. Accessed July 20, 2019

23. Martin BC, Wiley-Exley EK, Richards S, et al. Contrasting measures of adherence with simple drug use, medication switching, and therapeutic duplication. Ann Pharmacother 2009;43:36-44.

24. Quan H, Sundararajan $V$, Halfon $P$, et al. Coding algorithms for defining comorbidities in ICD-9-CM and ICD-10 administrative data. Med Care 2005;43:1130-1139.

25. Garg RJ, Kantarjian H, O'Brien $\mathrm{S}$, et al. The use of nilotinib or dasatinib after failure to 2 prior tyrosine kinase inhibitors: long-term follow-up. Blood 2009;114:4361-4368.

26. Giles FJ, Abruzzese E, Rosti G, et al. Nilotinib is active in chronic and accelerated phase chronic myeloid leukemia following failure of imatinib and dasatinib therapy. Leukemia 2010;24:1299-1301.

27. Ibrahim AR, Paliompeis $C$, Bua $M$, et al. Efficacy of tyrosine kinase inhibitors (TKIs) as third-line therapy in patients with chronic myeloid leukemia in chronic phase who have failed 2 prior lines of TKI therapy. Blood 2010;116:5497-5500.

28. Khoury HJ, Cortes JE, Kantarjian HM, et al. Bosutinib is active in chronic phase chronic myeloid leukemia after imatinib and dasatinib and/or nilotinib therapy failure. Blood 2012;119:3403-3412.

29. Russo D, Martinelli G, Malagola M, et al. Effects and outcome of a policy of intermittent imatinib treatment in elderly patients with chronic myeloid leukemia. Blood 2013;121:5138-5144.

30. Russo Rossi A, Breccia M, Abruzzese E, et al. Outcome of 82 chronic myeloid leukemia patients treated with nilotinib or dasatinib after failure of two prior tyrosine kinase inhibitors. Haematologica 2013;98:399-403.

31. Milojkovic D, Apperley J. Mechanisms of resistance to imatinib and second-generation tyrosine inhibitors in chronic myeloid leukemia. Clin Cancer Res 2009;15:7519-7527

32. West SL, Savitz DA, Koch G, et al. Recall accuracy for prescription medications: self-report compared with database information. Am J Epidemiol 1995;142:1103-1112.

33. White E, Armstrong BK, Saracci R. Principles of Exposure Measurement in Epidemiology: Collecting, Evaluating, and Improving Measures of Disease Risk Factors, 2nd ed. New York, NY: Oxford University Press; 2008 https://doi.org/10.1093/acprof:oso/9780198509851.001.0001

34. Dusetzina SB, Winn AN, Abel GA, et al. Cost sharing and adherence to tyrosine kinase inhibitors for patients with chronic myeloid leukemia. J Clin Oncol 2014;32:306-311.

35. Dusetzina SB. Drug pricing trends for orally administered anticancer medications reimbursed by commercial health plans, 2000-2014. JAMA Oncol 2016;2:960-961. 
Supplemental online content for:

\section{Long-Term Patterns of Oral Anticancer Agent Adoption, Duration, and Switching in Patients With CML}

Matthew P. Banegas, PhD, MPH; Donna R. Rivera, PharmD, MSc; Maureen C. O'Keeffe-Rosetti, MS;

Nikki M. Carroll, MS; Pamala A. Pawloski, PharmD; David C. Tabano, PhD; Mara M. Epstein, ScD;

Kai Yeung, PharmD, PhD; Mark C. Hornbrook, PhD; Christine Lu, MSc, PhD; and Debra P. Ritzwoller, PhD

J Natl Compr Canc Netw 2019;17(10):1166-1172

eTable 1: Logistic Regression Showing Factors Associated With Use of Oral Anticancer Agents 


\section{eTable 1. Logistic Regression Showing Factors Associated With Use of Oral Anticancer Agents ${ }^{a, b, c}$}

Factors

OR $(95 \% \mathrm{Cl})$

$P$ Value $^{\text {d }}$

Age at diagnosis

$0.96(0.95-0.97)$

$<.0001$

Female

$1.02(0.69-1.50)$

.9306

White

$1.26(0.74-2.13)$

.3966

Non-Hispanic

$0.75(0.35-1.62)$

.4671

Year of CML diagnosis

$1.16(1.10-1.21)$

$<.0001$

Charlson-Deyo comorbidity score

$0.87(0.76-0.99)$

.0385

Receipt of hydroxyurea

Abbreviations: $\mathrm{CML}$, chronic myeloid leukemia; $\mathrm{OR}$, odds ratio.

${ }^{a}$ Adjusted for age at diagnosis (continuous), female (vs male), white (vs nonwhite), non-Hispanic (vs Hispanic), year of CML diagnosis (continuous), Charlson-Deyo comorbidity score (continuous), receipt of hydroxyurea (vs no hydroxyurea received), and study site (results not shown).

bOral agents included bosutinib, chlorambucil, dasatinib, ibrutinib, imatinib, lenalidomide, nilotinib, ponatinib, ruxolitinib, and thalidomide. Nonoral agents included asparaginase, azacytidine, bacille Calmette-Guérin, bendamustine, bevacizumab, busulfan, cetuximab, cyclophosphamide, cytarabine, daunorubicin, decitabine, docetaxel, doxorubicin, etoposide, fludarabine, fluorouracil, gemcitabine, idarubicin, interferon alfa-2a, interferon alfa-2b, interferon beta-1a, melphalan, mitoxantrone, pegaspargase, pentostatin, rituximab, tocilizumab, topotecan, and vincristine.

cTable estimates based on 853 patients diagnosed with CML between 2000-2016 who were enrolled health plan members and who received care at 1 of

10 integrated health systems (Kaiser Permanente Colorado, Kaiser Permanente Northern California, Kaiser Permanente Northwest, Kaiser Permanente Hawaii, Kaiser Permanente Washington, Reliant Medical Group/Meyers Primary Care Institute, Marshfield Clinic Health System, Geisinger Health System, Henry Ford Health System, and HealthPartners Institute).

dBold indicates statistically significant $P$ value. 